\title{
The Education Management Information System: a key element to effectively monitor and evaluate a country's educational strategies
}

\author{
Daniel BÖJTE, PhD \\ Bucharest University of Economic Studies
}

\begin{abstract}
A properly designed and implemented Educational Management Information System (EMIS) assures precise, fluid and timely datasets which can be effectively used to monitor and evaluate national strategies. Data needs to be easily interpreted and a powerful EMIS is able to provide tools to extract the insights required by the policy makers and decision takers. As it is not only an IT tool or a database, but rather a multi-faceted framework that includes technological and institutional provisions for information collection, processing, and dissemination in an educational scheme, an EMIS is vital for monitoring changes, ensuring data quality and prompt reporting of significant planning and management information. The expanded scope of the fourth Sustainable and Development Goal within the UN's 2030 Agenda pushes EMIS to go far beyond administrative information, to information that can inform long-term instructional outcomes policies. EMIS should also investigate the connection between academic results and other socio-economic development industries, such as poverty alleviation, health, and jobs. This paper highlights the role and the importance of an effective EMIS in monitoring and evaluating the Romanian national strategies in education as part of the country's effort to align to the international educational standards regarding the access, participation and quality.
\end{abstract}

Keywords: decision; framework; insights; quality; technology; 


\section{Introduction}

Access to the use of the European Structural and Investment Funds (FESI) during the 20142020 programming period has involved a number of changes compared to the previous programming period. In this context, ex ante conditionalities have been defined to ensure the necessary preconditions for the effective and efficient use of European Union support. "Investments in education, training, including vocational training for acquiring skills and lifelong learning", representing Thematic Objective 10 established for this programming period, should be available for co-financing from the European Social Fund and the European Fund of Regional Development only if the ex-ante conditionalities associated with this objective are met. Consequently, in order to access the European Structural and Investment Funds (FESI), four sectoral strategies have been elaborated and approved by the Romanian Government Decisions for the educational sector:

- National Strategy for reducing early school leaving

- National Strategy of vocational education and training

- National Strategy for Tertiary Education

- National Lifelong Learning Strategy.

All these documents have been validated by the European Commission, so the four conditions being considered fulfilled.

The current programming period of the ESI Funds began in 2014 and runs until 2020. In the context of the adoption and approval of the ex-ante conditionalities in 2015-2016 and the financing of measures starting with the second part of 2017, the effective use of funds started with a considerable delay. This situation has determined the need for urgent actions and decisions, permanent and active monitoring of the implementation of interventions, in order to correct and improve certain tendencies.

Therefore, there is a critical need of timely and accurate data, so the Romanian Educational Management Information System has an important role to deliver the needed information to properly monitor and evaluate these strategies.

\section{Quality data for monitoring and evaluation of the strategies}

\subsection{Challenges in the monitoring process}

The Technical Groups responsible for the elaboration of the first monitoring report have often had difficulties in obtaining data regarding the accomplishment of some of the activities because, although they were programmed and implemented, factual data regarding the implementation were not collected and centralized.

This problem is partly caused by the apparent "failure to assume" all the responsibilities involved in implementing the strategies. As a measure of mitigating these difficulties, both a more intense popularization of the Action Plans for the following years, as well as the early information of all the sources regarding the data needed to be collected, are needed. 
The list of monitoring indicators needs to be revised so that ambiguities are eliminated (by clear definition / revision of the definitions of indicators where necessary) and overlaps, correction of recording errors and precise data sources and rhythmicity of updating.

Collecting financial data has proven to be the most difficult of challenges. As it can be seen in the reports for each strategy, each of the projected measures (and subsequent activity / activities) are evaluated within descriptive files that present a wealth of detailed technical information, but also financial information. In most cases, however, the disaggregation of information on institutional spending was impossible. As a consequence, in the description sheets, most of the financial information is not specified, the cause being the impossibility of disaggregating the expenses according to measures or actions. Therefore, for this monitoring stage, it was necessary to refer to the overall costs, about which information can be collected.

At this time, part of the strategy indicators cannot be collected, either because the required information is not reported in any database, or because the interoperation of existing databases is not completed. Another impediment was the difficulty of collecting the data, as well as the security of their accuracy.

\subsection{How can an a properly rolled-out EMIS help in monitoring and evaluation}

An EMIS's success depends upon three factors (Hua and Herstein, 2003):

- Timeliness and reliability: Timely and reliable production of data and information

- Integration and sharing: Data integration and data sharing among departments

- Effective usage: Effective use of data and information for educational policy decisions.

Effective EMIS provides useful information for procedures of a) management and administration; b) policy formulation; c) tracking and assessment, which practically is the monitoring and evaluation. These procedures are interconnected and take place in educational systems, from central to local and within educational organizations themselves, at different places. (UNESCO, 2018).

\subsubsection{Timeliness and reliability}

Up-to-date data and information development require that all prospective data and information manufacturers or customers have mutual knowledge of the following:

1) EMIS data generated frequently must satisfy the requirements of the general instructional planning and budgeting process.

2) Regularly generated EMIS information must satisfy the requirements of educational services such as the Logistics Unit and other classroom supply systems.

3) EMIS information generated regularly must fulfil the requirements of instructional surveillance and assessment, as well as timely policy research and guidelines.

4) Regularly generated EMIS information must satisfy the requirements of global cooperation and communication.

The timeliness within the Ministry of Education to meet these requirements is crucial to the achievement of EMIS growth. The method of data collection, data entry, data processing, data integration, data analysis, and data reporting should be brief, effective, and productive in order to ensure the prompt output of data and information to satisfy these requirements. This can 
often be accomplished by raising the amount of effort, starting previous preparations, proposing and enhancing task deadlines, institutionalizing EMIS as a routine management process, and enhancing EMIS data-related operations coordinating ability.

The general layout of the information collection method, information instrument development, and computer database application design and development must be thoroughly designed to increase data reliability. No quantity of technological innovation can improve from initial information of lousy quality.

In data and information management, "Garbage in, garbage out" is as valid as it is in computer programming. Timeliness and reliability can influence the amount of confidence and trust in the data of the information consumer. Delay in information manufacturing and unreliable information output can readily lead to a lack of frustration in information use and management, leading in inadequate planning and budgeting, tracking and assessment, policy analysis, and decision making.

When consumers of data and information (e.g., policy makers, analysts) lose faith in the capacity or credibility of EMIS, they often prevent assistance to maintain, reinforce, and upgrade the EMIS scheme. In turn, manufacturing capacity for data and information becomes even worse or decreased, further jeopardizing the ability to generate timely and reliable data. It is necessary to eradicate this vicious cycle that permeates specific instructional systems. A good culture of decision-making and governance based on information must be nurtured and created, allowed, and endorsed by an information-user-demand-driven EMIS (Hua and Herstein, 2003).

\subsubsection{Integration and sharing}

Integration of data is one of the most significant approaches for EMIS growth. It implies multi-source information (payroll, accomplishment, school census), multi-year, and multilevel information (student, teacher, or school level) can be connected, incorporated, or combined. Data integration aims to add value to the information already gathered and accessible within the same scheme in different scattered locations. Data integration is a must before conducting a high-level and high-quality policy analysis or planning exercise by an instructional policy analyst or planner. It is prevalent to see multiple units collecting and managing big databases within a Ministry of Education and not sharing them. These different information sets are gathered to define certain system aspects. For instance, in a Ministry of Education, information on student accomplishment is often gathered, managed, and accessible in an exam unit; teacher qualification and wage information are in a payroll office; information on enrolment and school inputs are in a statistical unit, and information on textbook supplies, classroom equipment, and other teaching resources are often in a supply office. For their task planning and management, these offices often have distinct databases and rarely share them with other offices. These various information sets are often intended in distinct apps for databases, arranged in distinct platforms, and coded with a self-developed identification code. As a result, unless a data integration strategy is implemented, data cannot be easily incorporated or used like this.

There can be no monitoring and assessment system, a planning and policy analysis system, or an efficient and policy-relevant EMIS system without coordinated leadership. Without such schemes, policy investigations such as: how much teacher qualification and salary contribute 
to student learning accomplishment, given that the classroom climate and distribution of resources are the same? What is the effect on student learning success of a brand-new teacher training program or curriculum (or any recent instructional investment project)?

We need to incorporate multi-source information so we can do the correct data analysis to answer the correct policy questions. Once centrally embedded and structured, multi-level information from various sources and years could have tremendous importance for policyrelevant research and evaluation and education leadership enhancement (Hua and Herstein, 2003).

\subsubsection{Effective usage}

An institutional culture of making policy choices based on data and information is one of the most critical variables contributing to the achievement of EMIS growth. This culture is a userdemand-enabling environment in which it is possible to build, strengthen, and further develop the ability for policy studies and analysis. Data and information consumers are policy makers, planners, policy analysts, and other significant stakeholders. The demand for data and information use should foster and nurture the remarkable growth of a culture of decisionmaking based on the information and the EMIS scheme. The institutional demand for data and information use is often converted into or proved through the ability of the Departmental Monitoring and Evaluation Unit, Policy Research and Analysis Unit, and Budgeting and Planning Unit. A weak capability would hurt the growth of EMIS in any of these leadership units.

\subsection{EMIS as a solution}

\subsubsection{The Romanian EMIS component for pre-university education}

The Romanian Ministry of National Education successfully implemented, at the end of 2015, one of its EMIS component designed for the pre-primary, primary and secondary education system. The IT system called SIIIR provides full management services for the activities of the pre-university educational system from an operational, technical, administrative and strategic point of view. It addresses both the needs of the users at central level (mainly related to the decision making based on the analysis of the performance indicators, but also to ensure the transparency of the investment processes, the management of the human resources problem), as well as the needs identified at local level (management of activities, processes and flows at the level of the educational unit).

In 2016, The World Bank assessed SIIIR as one of the Romanian EMIS main component. The Systems Approach for Better Education Results (SABER) is an established tool designed to support countries in systematically examining and strengthening the performance of their education systems.

Part of the World Bank's Education Sector Strategy, SABER uses diagnostic tools for examining education systems and their component policy domains against global standards and best practices and in comparison, with the policies and practices of countries around the world. By leveraging this global knowledge, the SABER tools fill a gap in the availability of data and evidence on what matters most to improve the quality of education and achievement of better results. This report discusses the results of applying the SABER-Education Management Information Systems (EMIS) tool in Romania. The objectives of this report are 
to examine the system according to key policy areas, identify successes and challenges in the system, and provide recommendations to support the continued advancement of the EMIS in Romania. 


\begin{tabular}{|c|c|}
$\begin{array}{c}\text { Policy Areas } \\
\text { Enabling } \\
\text { Environment }\end{array}$ & $\begin{array}{l}\text { Policy Levers: legal framework, organizational structure } \\
\text { and institutionalized processes, human resources, } \\
\text { infrastructural capacity, budget, data-driven culture }\end{array}$ \\
\hline $\begin{array}{c}\text { System } \\
\text { Soundness }\end{array}$ & $\begin{array}{l}\text { Policy Levers: data architecture, data coverage, data } \\
\text { analytics, dynamic system, serviceability }\end{array}$ \\
\hline Quality Data & $\begin{array}{l}\text { Policy Levers: methodological soundness, accuracy and } \\
\text { reliability, integrity, periodicity, and timeliness }\end{array}$ \\
\hline $\begin{array}{c}\text { Utilization for } \\
\text { Decision Making }\end{array}$ & Policy Levers: openness to EMIS users, operational use, \\
\hline
\end{tabular}

Source: Abdul-Hamid, 2014

The SABER Country Report 2016 (World Bank, 2016) brief findings are as follows:

a) Enabling environment: Ministerial Order 5760/2006 established the National Education Database (BDNE), which became the mandatory system for education data collection in 2006. BDNE later was transformed into the Integrated IT System of Romanian Education (SIIIR), which is currently used for all data collection, validation, and analysis. A recently signed agreement between the Ministry of National Education and Scientific Research (MNESR) and the National Institute for Statistics (NIS) has fully institutionalized SIIIR as the sole provider of education data in data collection, validation, processing, and dissemination, also to be directly integrated with the tertiary education sector by 2017. In accordance with the Confidentiality and Data Privacy Law 677/2001, the Romanian Data Protection Agency conducted an extensive data protection audit in 2008. It has since established EMIS compliance with data protection laws, defining responsibilities, roles, and guidelines for the EMIS. EMIS staffing, however, is not yet institutionalized. The hiring and retention of highly qualified and skilled human resources remain a substantial challenge and threat to the sustainability of the EMIS. EMIS positions tend to lack competitiveness and appeal in comparison with the private sector. Moreover, no institutionalized professional development and training plans have been set forth by the MNESR. 
b) System soundness: EMIS architecture and design establish the functionality and operability of the Romanian EMIS. The system is able to produce quality and timely data but is not yet fully integrated. The EMIS would benefit from smart and dynamic features such as feedback mechanisms to bridge the information gap between data providers and data users. A fully integrated early warning system would enable teachers, parents, and school administrators to identify students at risk and ensure timely intervention to improve learning outcomes. Additional analytics features of the EMIS may include projections (e.g., student population), what-if analysis, and data associations. To ensure full data coverage, particular importance should be paid to socially disadvantaged groups, such as Roma, because the system currently fails to collect data on them. The EMIS is web-based and has been customized employing a Business Intelligence software.

c) Quality data: Data collected by the Romanian EMIS are comprehensive but would benefit from improved accuracy and reliability measures. The data collected cover a wide range of important indicators in administrative, human resource, financial, and learning outcome data. To ensure timely annual statistics reports, data compliance policies and penalties for schools have been put into place. However, the education indicators are often not clearly defined, causing conflicting results in, for example, reporting of dropout rates. The initial data collection and entry are carried out by administrative staff, who would benefit from additional instruction and systematic training to ensure accurate and reliable data (e.g. on school infrastructure). School inspectorates are tasked to track data production processes to certify that best practice standards are employed.

d) Utilization for Decision Making: Despite comprehensive and quality data, the EMIS remains closed and underutilized. The system does not foster data access, sharing, and utilization. The establishment of SIIIR has improved data sharing, transparency, and access to a certain degree, but more efforts should be made, and utilization of the available information remains challenging. Policy makers do not yet utilize data for decision making and could benefit from sharing data with research agencies to design education policies. Currently an integrated data utilization system is not in place for professional development and continuous training for teachers and EMIS staff. Moreover, even though schools, teachers, and parents are given access to education data in the form of national reports, they are often unable to use the data effectively. Once the Electronic National Grade-book System is established and operational, it will enable schools to make use of the data collected by the EMIS in the context of informed decision making to improve learning outcomes of students.

\subsubsection{The Romanian EMIS component for higher education}

The Romanian Ministry of National Education, as the central authority that coordinates the national education system, is responsible for evaluating it based on criteria and standards, based on which it defines the actions, strategies and programs for the entire education system in Romania, including the Higher Education System.

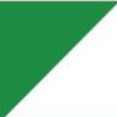


At this moment, The Ministry of Education has a set of non-integrated information, databases and primary tools for analysis and control, thus the need to ensure an integrated system, at national level, that offers an overview of the key aspects of the higher education system.

Given the large number and variety of activities coordinated by the Ministry of National Education regarding the process of managing the financing of Higher Education, there is an acute need to reorganize the institutional processes of monitoring and reporting, by creating a specialized system, based on the success of the SIIIR EMIS component presented above.

The main problems identified, which make it necessary to implement such a system, are related to the impossibility of automating the processes of transmission, validation, centralization of data and the modalities of establishing the indicators regarding the Higher Education system. Therefore, it is necessary to generate new working procedures, as well as optimizing the existing flows where appropriate.

According to the Country Report prepared for the Conference of Ministers of Education, signatories to the Bologna Declaration, the number of study programs that allow students to choose from a wide range of optional courses has increased steadily in recent years. Students can choose a modular course of study, and within the same module they can choose from a wide range of disciplines. Romania has made important steps towards the European Higher Education Area, by reorganizing the entire spectrum of university programs. A new structure of higher education was adopted by Law 288/2004 on the organization of university studies, which provides the legislative framework for the introduction of the three cycles: undergraduate, master's and doctoral studies, according to the Bologna Process objectives. The law entered into force in the academic year 2005-2006 and the first generation of graduates was that of 2008/2009.

The problems identified are therefore of a methodological nature, they are current, and their solution represents a priority for the Ministry of National Education as the component of the higher education structure reform in Romania, which is currently underway and aims at increasing student mobility, increasing their chances in the labor market, reducing the number of university specializations and increasing participation in master and doctoral programs.

The implementation of the EMIS component for Higher Education ensures the development of the institutional capacity of the Ministry, by carrying out studies on the existing legislative framework and generating new unified working processes, as well as by identifying modern tools to support the activities for managing the budget of the Romanian universities and uniformizing the data from the system.

That is why, in 2014, the Ministry of National Education started the implementation of a European funded project called "Improving the organizational efficiency of the Higher Education system by establishing a set of specific indicators and creating the management and monitoring capability" (also known as "The Higher Education Database" - "BDNE").

The general objective of the project consisted in the optimization and consolidation of the institutional processes of monitoring and reporting carried out by the Ministry of National Education through the implementation of an adequate information system.

As specific objectives, the project aimed to:

- Increasing the efficiency of the process of managing the financing of the higher education system by carrying out a complete set of procedures, methodologies and analysis documents; 
- Developing an automated system for allocating funds based on the criteria imposed by the relevant legislation in the field - mainly the National Education Law no. 1/2011, Order no. 3998/2012 of 05/05/2012 and Law 288/2004, as subsequently amended and supplemented;

- Efficiency of the reporting system necessary for granting the financing in the higher education system, by implementing at the ministry level a centralized system of transmission, retrieval and management of the reports;

- Increasing the capacity for analysis and synthesis of the Ministry regarding the financing of the higher education system by developing and implementing a tool for automatic generation of real-time reports on the centralized situation of the bachelor, master and doctorate cycles at each accredited university;

- Increasing labor productivity by providing training courses for staff involved in financing the higher education system.

Thus, BDNIS, as an integrated information system, is meant to provide a unified procedural framework for the management of the financing of the Higher Education System, to define a set of specific indicators and ensure their control and monitoring facilities, data storage and long-term archiving.

Also, the system ensures the increase of the degree of transparency and control over the processes, but also a better implementation and application of the legislation in the field. The implementation of this EMIS component ensures an adequate infrastructure for the activity of the Ministry for the management of the higher education system, thus being possible to improve the regulatory mechanisms and to create a unified institutional framework regarding the financing of the mentioned field.

BDNIS is not only an operational, technical and administrative instrument, but also a strategic instrument that contributes to the elaboration of public policies, the education strategy and the efficiency of communication with the citizens and institutions under subordination and coordination. Also, it is the integration platform for all the non-integrated information systems that are already used.

Currently, this system is operational but it is not rolled out yet because of several legislative issues regarding the universities' autonomy. If the system should have been rolled out by now, the challenges aforementioned should have been addressed.

\section{Conclusion}

One of the most critical factors that contributes to the success of the EMIS development is an institutional culture of making policy decisions based on data and information. This culture is a user-demand-enabling environment under which the policy research and analysis capacity can be built, strengthened, and further developed. Policy makers, planners, policy analysts, and other high stakeholders are the users of the data and information. The demand for using data and information should stimulate and nurture the healthy development of an informationbased decision-making culture and the EMIS system. Often, the institutional demand for use of data and information is translated into or demonstrated by the capacity of the Monitoring and Evaluation Unit, Policy Research and Analysis Unit, and Budgeting and Planning Unit 
within the Ministry. A weak capacity in any of these management units would exert a negative impact on EMIS development.

It is very important to understand that EMIS development is not IT development. No one wants just "policy talk." Policy must be supported by evidence or analysis of the evidence. EMIS is developed to provide that evidence. EMIS and other management functions such as monitoring and evaluation, policy research and analysis, and budgeting and planning together should provide the results of the analyses of that evidence. This is the support that Ministries of Education need and policy makers in the Ministries of Education deserve (Hua and Herstein, 2003).

\section{References}

[1] Abdul-Hamid, H. 2014, What Matters Most for Education Management Information Systems: a Framework Paper, World Bank, [Online] Available at http://wbgfiles.worldbank.org/documents/hdn/ed/saber/supporting_doc/Background/EMIS /Framework_SABER-EMIS.pdf

[2] Hua, H. and Herstein, J., 2003, Education management information system (EMIS): Integrated data and information systems and their implications in educational management. In Annual conference of comparative and International Education Society, [Online] Available at http://www.infodev.org/infodevfiles/resource/InfodevDocuments_188.pdf.

[3] UNESCO, 2018, Re-orienting Education Management Information Systems (EMIS) towards inclusive and equitable quality education and lifelong learning, [Online] Available at http://unesdoc.unesco.org/images/0026/002619/261943e.pdf

[4] World Bank, The, 2016, SABER EMIS Romania Country Report, [Online] available at http://wbgfiles.worldbank.org/documents/hdn/ed/saber/supporting_doc/CountryReports/E MS/SABER_EMIS_Romania_Report2016.pdf 